\title{
Allopurinol use and the risk of cataract formation
}

\author{
WALTER K CLAIR, LEO T CHYLACK JR, E FRANCIS COOK, \\ AND LEE GOLDMAN
}

From the Departments of Medicine and Ophthalmology, Brigham and Women's Hospital and Harvard Medical School, Boston, Massachusetts, USA

SUMMARY Several reports have suggested an association between chronic allopurinol ingestion and cortical and subcapsular cataract formation. To examine this possibility we identified 51 allopurinol users and compared their lenses with those of 76 patients who did not use allopurinol. The existence of lens opacities and the level of visual acuity were assessed by review of medical records or by prospective ophthalmic examinations; in both phases of the study the examiners were blinded as to the patient's use or non-use of allopurinol. Three different outcomes were considered: formation of any cataract, formation of a posterior subcapsular cataract, and formation of a cataract contributing to a corrected visual acuity of 20/30 or worse. The risk ratio for the formation of any cataract was $1.3(95 \%$ confidence interval: $0 \cdot 8,2 \cdot 0)$, the risk ratio for the formation of a posterior subcapsular cataract was $0.9(0 \cdot 3,2 \cdot 0)$, and the risk ratio for the formation of a cataract contributing to a loss of visual acuity was $1.3(0.6,2 \cdot 9)$. None of these risk ratios was changed appreciably after controlling for age, sex, hypertension, or diabetes. Thus, after a mean of 6.9 years of allopurinol use, we found no evidence to confirm that allopurinol users were at higher risk of acquiring cataracts.

Cases reported to the National Registry of DrugInduced Ocular Side Effects ${ }^{1}$ have suggested an association between allopurinol use and cortical and subcapsular cataract formation. Lerman et al. ${ }^{2}$ demonstrated that allopurinol is photobound in the human lens and suggested that it is cataractogenic in patients in whom this photobinding has occurred. Although Jick and Brandt ${ }^{3}$ found no association between allopurinol use and cataract surgery, their study did not include ophthalmic examinations. To find out whether allopurinol use increases the risk of cataract formation we designed a study that included ophthalmic examinations.

\section{Patients and methods}

RETROSPECTIVE PHASE

From hospital pharmacy records we identified 457 allopurinol users without concomitant chemotherapy, including 56 who had received an ophthalmic examination at our institution. After excluding

Correspondence to Lee Goldman, MD, Department of Medicine, Brigham and Women's Hospital, 75 Francis Street, Boston, MA 02115, USA. patients whose examinations preceded allopurinol use, or whose records suggested a history of congenital cataracts, ocular disease, ocular trauma, or exposure to toxic cataractogenic agents $(n=26)$, or whose examinations did not include a lens examination $(n=6)$, we had 24 allopurinol users for this phase of the study. By the same exclusion criteria we identified 54 allopurinol non-users from among all patients who had been referred for ophthalmic examination prior to the initiation of amiodarone or hydroxychloroquine therapy.

PROSPECTIVE PHASE

Of the 401 allopurinol users without ophthalmic examinations in the retrospective review 193 who were known to be alive and in the proximity of our hospital were invited to receive a free examination: 159 declined, six did not keep a scheduled appointment, and one was excluded by the same criteria used in the retrospective phase, leaving 27 allopurinol users in the prospective phase. Ophthalmic examinations to assess visual acuity and lens opacities were also performed on 22 non-users of allopurinol who were recruited from our hospital based internal medicine practice. 
BASELINE INFORMATION

By medical record review (retrospective phase) or patient interview (prospective phase) we obtained each patient's sex; age at the ophthalmic examination; history of diabetes, hypertension, or glaucoma; use of birth control pills, oestrogens, miotic medications, or phenothiazines; and occupation. No records or interviews suggested congenital cataracts, ocular disease, ocular trauma, or exposure to cataractogenic agents.

OPHTHALMIC EXAMINATION INFORMATION

From the medical records in the retrospective phase and the detailed ophthalmic examinations in the prospective phase we recorded each patient's initial, corrected, and best corrected Snellen visual acuity; nuclear colour as pale yellow, yellow, dark yellow, or brown/black; existence or previous removal of cataracts; evidence of injury or inflammation; and presence of retinopathy (diabetic retinopathy or maculopathy).

Cataracts were divided into four major classesnuclear, cortical, supranuclear, and posterior subcapsular. Age related changes such as cortical flecks and isolated vacuoles, lamellar separations, and sutural prominence were not classified as cataracts, nor were Mittendorf dots. If the cortical, supranuclear, or subcapsular opacity was visible at the slitlamp against the red reflex, and if it consisted at least of linear or clustered aggregates of the flecks, vacuoles, and lamellar separations, it was designated a cataract. Patients with a best corrected Snellen visual acuity of $20 / 30$ or worse as a result of retinal disease were excluded. Retrospective information was obtained from medical records without any knowledge of allopurinol use. Similarly, information obtained from all prospective ophthalmic examinations was determined without knowledge of allopurinol use.

Three aspects of cataract existence were considered as possible outcomes: the development of any cataract, posterior subcapsular cataracts, or cataracts that contributed to a best corrected visual acuity of $20 / 30$ or worse. Because of inadequate medical record information, five allopurinol users and four non-users in the retrospective phase could not be classified as to their best corrected visual acuity and were eliminated in the analyses for this outcome.

\section{STATISTICAL METHODS}

All numbers and analyses were based on patients, not individual eyes. Estimated risks for cataract development were compared by the $\chi^{2}$ test. ${ }^{4}$ Risk ratios and corresponding $95 \%$ confidence intervals were constructed. ${ }^{5}$ In the one instance where an estimated risk
Table 1 Distribution of age, sex, diabetes, and hypertension for allopurinol users and non-users

\begin{tabular}{llc}
\hline & $\begin{array}{l}\text { Allopurinol users } \\
(n=51)\end{array}$ & $\begin{array}{l}\text { Non-users } \\
(n=76)\end{array}$ \\
\hline Age & & \\
Mean (years) & $61 \cdot 8$ & $57 \cdot 6$ \\
$\quad$ Standard deviation & $12 \cdot 4$ & $12 \cdot 2$ \\
Male sex & $38(75 \%)$ & $27(34 \%)$ \\
Diabetes & $13(25 \%)$ & $5(7 \%)$ \\
Hypertension & $27(53 \%)$ & $26(34 \%)$ \\
\hline
\end{tabular}

for non-users equalled $0 \cdot 0,0 \cdot 5$ was added to the numerator of this risk. ${ }^{5}$ Potential confounding by age, sex, diabetes, and hypertension was controlled by two methods: a logistic regression model that expressed each outcome as a function of allopurinol use and the potential confounders ${ }^{5}$; and a propensity score analysis, ${ }^{6}$ by which patients were stratified by their likelihood for allopurinol use, according to subranges of a logistic regression model that expressed allopurinol use as a function of the confounders. Power calculations for detecting relevant values for the risk ratio were based on the non-null distribution of the test statistic in the usual $\chi^{2}$ test of association. ${ }^{4}$ Increased risk for cataract formation with increased duration of allopurinol use was examined by a $\chi^{2}$ test for trend.?

\section{Results}

Allopurinol users were older and contained more males, more diabetics, and more hypertensives than non-users (Table 1). Estimated risk ratios were similar for the two phases of the study, except for the formation of a posterior subcapsular cataract (Table 2 ), where these estimated risk ratios had the widest confidence intervals. Therefore, because of the strong similarities of results in the two data sets, all further analyses were based on the single combined data set.

Compared with non-users the allopurinol users did not appear to have an excess risk for any of the three outcomes. Adequate data on the exact duration of allopurinol use were available for 37 of the 51 allopurinol users. The mean duration of use was 6.9 years, standard deviation 6.9 years. Only seven patients had used allopurinol for more than 10 years. No tendency for increased risk of cataract formation with increased duration of allopurinol use was observed for any of the three outcomes. When potential confounding by age, sex, diabetes, and hypertension was controlled both by logistic regression and by propensity score analyses, the adjusted risk ratios remained non-significant and were very similar to the crude estimates. Our sample size is 
Table 2 Estimated risks and risk ratios for the formation of any cataract, of a posterior subcapsular cataract, or of a cataract contributing to loss of visual acuity* for allopurinol users compared with non-users in retrospective, prospective, and combinęd data sets

\begin{tabular}{|c|c|c|c|c|c|c|c|}
\hline \multirow[t]{2}{*}{ Data set } & \multirow{2}{*}{$\begin{array}{l}\text { Number of } \\
\text { subjects }\end{array}$} & \multicolumn{2}{|c|}{ Any cataract } & \multicolumn{2}{|c|}{ Posterior subcapsular cataract } & \multicolumn{2}{|c|}{$\begin{array}{l}\text { Cataract leading to loss of } \\
\text { visual acuity }\end{array}$} \\
\hline & & Risk & Risk ratio & Risk & Risk ratio & Risk & Risk ratio \\
\hline \multicolumn{8}{|l|}{ Retrospective } \\
\hline Users & 24 & $0 \cdot 33$ & \multirow{2}{*}{$1 \cdot 1(0 \cdot 5,2 \cdot 1) \dagger$} & $0 \cdot 17$ & \multirow{2}{*}{$0.9(0 \cdot 3,2 \cdot 6) \dagger$} & $0 \cdot 32$ & \multirow{2}{*}{$1 \cdot 6(0 \cdot 7,3 \cdot 8) \dagger$} \\
\hline Non-users & 54 & $0 \cdot 31$ & & $0 \cdot 19$ & & $0 \cdot 20$ & \\
\hline \multicolumn{8}{|l|}{ Prospective } \\
\hline Users & 27 & $0 \cdot 52$ & \multirow{2}{*}{$1 \cdot 3(0 \cdot 7,2 \cdot 4)$} & $0 \cdot 04$ & \multirow{2}{*}{$2 \cdot 5(0 \cdot 3,21 \cdot 6)$} & $0 \cdot 11$ & \multirow{2}{*}{$2 \cdot 4(0 \cdot 3,20 \cdot 4)$} \\
\hline Non-users & 22 & $0 \cdot 41$ & & $0 \cdot 00$ & & $0 \cdot 05$ & \\
\hline \multicolumn{8}{|l|}{ Combined } \\
\hline Users & 51 & 0.43 & \multirow{2}{*}{$1 \cdot 3(0 \cdot 8,2 \cdot 0)$} & $0 \cdot 10$ & \multirow[t]{2}{*}{$0.9(0 \cdot 3,2 \cdot 0)$} & $0 \cdot 20$ & \multirow[t]{2}{*}{$1 \cdot 3(0 \cdot 6,2 \cdot 9)$} \\
\hline Non-users & 76 & $0 \cdot 34$ & & $0 \cdot 13$ & & $0 \cdot 15$ & \\
\hline
\end{tabular}

*Cataract contributing to corrected visual acuity $20 / 30$ or worse. $† 95 \%$ Confidence interval.

sufficient for detecting large effects of allopurinol on all three outcomes (for example, $\mathrm{RR}=3 \cdot 0$ ) and small effects (for example, $R R=1 \cdot 5$ ) for any cataract (Table 3 ) but insufficient for detecting small effects for posterior subcapsular cataracts or cataracts contributing to a best corrected visual acuity of $20 / 30$ or worse.

\section{Discussion}

March et al. ${ }^{8}$ demonstrated that oxipurinol, an allopurinol metabolite, enters the human lens. Using high-resolution phosphorescence spectroscopy, Lerman et al. ${ }^{24}$ showed that allopurinol is photobound in the human lens. They have suggested that this leads to photosensitisation and subsequent cataract formation. Our study differs from the report by Jick and Brandt, ${ }^{3}$ who found no association between allopurinol use and surgery for cataract extraction, because our patients had full ophthalmic examinations by methods shown to be valid, reproducible, and sufficiently sensitive.

We did not find evidence of a major increase in risk

Table 3 Power of test of significance for our data set to detect risk ratios of $1 \cdot 5,2 \cdot 0$, and 3.0 for the formation of any cataract, of: a posterior subcapsular cataract, or of a cataract contributing to loss of visual acuity*

\begin{tabular}{llll}
\hline $\begin{array}{l}\text { Risk ratio to } \\
\text { be detected }\end{array}$ & Any cataract & $\begin{array}{l}\text { Posterior } \\
\text { subcapsular } \\
\text { cataract }\end{array}$ & $\begin{array}{l}\text { Cataract } \\
\text { contributing } \\
\text { to loss of } \\
\text { visual acuity }\end{array}$ \\
\hline 1.5 & 0.52 & 0.17 & 0.19 \\
2.0 & 0.97 & 0.46 & 0.50 \\
3.0 & 0.99 & 0.92 & 0.94 \\
\hline
\end{tabular}

*Cataract contributing to corrected visual acuity of $20 / 30$ or worse. for any of three types of cataracts among users of allopurinol. One potential problem is possible misclassification of the presence or absence of a cataract in our retrospective phase. However, all determinations of outcome obtained from medical records were obtained without knowledge of allopurinol use, and therefore any misclassification would be expected to be random, that is, independent of allopurinol use. The strong similarities in results from both study phases, despite different methods for detecting outcomes, makes it unlikely that there was any substantial random misclassification bias, especially since the prospective phase strictly followed a valid and reproducible classification system..$^{10-14}$

Another possible explanation for the negative results is a selection bias by which either our allopurinol users or non-users do not reflect the true risks for cataract formation. For example, our nonusers with rheumatic conditions may have used aspirin, which may be protective against cataract formation. ${ }^{15}$ However, this potential bias would tend to overstate the association between allopurinol use and cataract formation rather than bias it towards the null value. Although our low recruitment rate could potentially introduce bias, we considered it was imperative to include a detailed ophthalmic examination to eliminate an ascertainment bias that might have existed in a study that relied on cataract surgery, ${ }^{3}$ rather than on the presence of a cataract. Although it is possible that our negative results are due to an insufficient period of allopurinol use, there was no increased risk for cataract formation with increased duration of allopurinol use.

Finally, our study has a limited ability to identify a true but relatively weak association. Nevertheless, the consistency of the results with those of the prior study ${ }^{3}$ argue against a strong association between allopurinol and cataracts. 
Dr Chylack is a recipient of Research to Prevent Blindness's senior scientific investigator award (1986) and the Alcon Research Institute award (1986). This work was supported in part by the Brigham Surgical Group Foundation.

\section{References}

1 Fraunfelder FT, Hanna C, Dreis MW, Cosgrove KW. Cataracts associated with allopurinol therapy. Am J Ophthalmol 1982; 94: $137-40$.

2 Lerman S, Megaw JM, Gardner K. Allopurinol therapy and cataractogenesis in humans. Am J Ophthalmol 1982; 94: $141-6$.

3 Jick H, Brandt DE. Allopurinol and cataracts. Am J Ophthalmol 1984; 98: 355-8.

4 Colton T. Statistics in medicine. Boston: Little, Brown, 1974: 163-81.

5 Kleinbaum DG, Kupper LL, Morgenstern H. Epidemiologic research: principles and quantitative methods. Belmont: Wadsworth, 1982: 320-456.

6 Rosenbaum PR, Rubin DB. The central role of the propensity score in observational studies for causal effect. Biometrika 1983; 70: 41-55.

7 Brown BW, Hollander M. Statistics: a biomedical introduction. New York: Wiley, 1977: 211-5.

8 March WF, Goren S, Shoch D. Action of allopurinol on the lens. In: Leopold IH, ed. Symposium on ocular therapy. St Louis: Mosby, 1974: 83-95.
9 Lerman S, Megaw JM, Fraunfelder FT. Further studies on allopurinol therapy and human cataractogenesis. Am J Ophthalmol 1984; 97: 205-9.

10 Chylack LT Jr. Classification of human cataracts. Arch Ophthalmol 1978; 96: 888-92.

11 Chylack LT Jr, Lee MR. Tung WH, Chang HM. Classification of human senile cataractous change by the American Cooperative Cataract Research Group (CCRG) Method: I. Instrumentation and technique. Invest Ophthalmol Vis Sci 1983: 24: 424-31.

12 Chylack L'T Jr, White O, Tung WH. Classification of human senile cataractous change by the American Cooperative Cataract Research Group (CCRG) Method: II. Staged simplification of cataract classification. Invest Ophthalmol Vis Sci 1984: 25: 16673.

13 Chylack LT Jr, Ranoil BJ, White O. Classification of human senile cataractous change by the American Cooperative Cataract Research Group Method: III. The association of nuclear color (sclerosis) with extent of cataract formation, age and visual acuity. Invest Ophthalmol Vis Sci 1984; 25: 174-80.

14 Chylack LT Jr, Rosner B, Garner W, et al. Validity and reproducibility of the Cooperative Cataract Research Group (CCRG) cataract classification system. Exp Eye Res 1985: 40: $135-47$.

15 Cotlier E, Sharma YR, Niven T, Brescia M. Distribution of salicylate in lens and intraocular fluids and its effect on cataract formation. Am J Med 1983; 74: 83-90.

Accepted for publication 14 April 1988. 